\title{
Prevalencia de malformaciones congénitas en recién nacidos de madres que residen en el estado de Aguascalientes. Perspectiva de los últimos 10 años
}

Palacios-Arenas Martín Oswaldo*, Terrones-Saldívar Ma del Carmen**

\section{Resumen}

- Introducción: las malformaciones congénitas (MC) son defectos es- tructurales, morfológicos, funcionales de uno o varios órganos o zonas - más extensas del organismo. La Organización Mundial de la Salud - (OMS) estima una prevalencia internacional de 3-5\%. Cifras nacio- nales 2010 ubican a Aguascalientes con la tasa más alta de MC - (I87/I0,000 nacimientos). Objetivo general: determinar la prevalen- cia de MC en recién nacidos de madres que residen en el estado de - Aguascalientes. Material y métodos: estudio observacional, retrospec-

- tivo y transversal. Universo: recién nacidos vivos de 22 a 42 semanas

- de gestación ylo con peso $\geq 500 \mathrm{~g}$ con diagnósticos de una o más

- MC registrados en el certificado de nacimiento entre enero 2008 a

- diciembre 2017. Resultados: se registraron 267,489 recién nacidos

- vivos. La prevalencia de MC fue 2.1\% (5595) con una tasa de 209.11

- 10,000 nacidos vivos. El sistema osteomuscular fue el más afectado

- (39.3\%). Individualmente, el testículo no descendido fue la MC más

- frecuente (I4.2\%). La prevalencia de MC por municipio de residencia

- presentó asociación estadísticamente significativa $(p<0.01)$, siendo

- El Llano (2.9\%) y Aguascalientes (2.4\%) los que presentaron mayor

- frecuencia de MC. Conclusión: la prevalencia de MC identificada fue

- menor a los estándares internacionales de 3\%, pero superior a los re-

- portes nacionales de $0.7 \%$. El sistema más afectado por MC fue simi-

- lar a reportes nacionales previos. Se debe interpretar cautelosamente

- los resultados por un posible subregistro en el sistema de certificados

- de nacimiento; sin embargo, es el instrumento reconocido por el INEGI

- para establecer las tasas de eventos perinatales. LUXMÉDICA AÑO 15,

- NÚMERO 43, ENERO-ABRIL 2020, PP 3-12

Palabras clave: malformaciones congénitas, salud reproductiva, salud medioambiental.

\section{Abstract}

Introduction: Congenital anomalies (CA) are structural, morphological, functional defects of one or more organs or larger areas of the organism. The WHO estimates an international prevalence of 3-5\%. National numbers from 2010 place Aguascalientes at the highest rate of CA (187// 0,000 births). Objective: To determine the prevalence of CA in newborns of mothers living in the state of Aguascalientes. Methods: This is an observational, retrospective, cross-sectional study. Universe: live newborns from 22 to 42 gestation weeks and/ or weighing greater than or equal to $500 \mathrm{gr}$ with diagnoses of one or more CA registered in the birth certificate between January 2008 - December 2017. Results: 267,489 live births were registered. The prevalence of Ca was 2. $1 \%$ (5595), with a rate of 209. I/ 0,000 live births. The musculoskeletal system was the most affected (39.3\%). Individually, the undescended testicle was the most frequent CA (14.2\%). The prevalence of CA by municipality of residence showed a statistically significant association ( $p<0.01$ ), with Llano $(2.9 \%)$ and Aguascalientes (2.4\%) having the most CA. Conclusion: Prevalence of CA was lower than the international standards of $3 \%$ but higher than the national reports of $0.7 \%$. The system most affected by CA was similar to previous national reports. The results should be cautiously interpreted since there could be a possible under-registration in the birth certificate system, however, it is the instrument recognized by INEGl to establish the rates of perinatal events. LUXMÉDICA AÑO 15, NÚMERO 43, ENERO-ABRIL 2020, PP 3-12

Keywords: congenital anomalies, reproductive health, environmental health

** Martín Oswaldo Palacios Arenas. Médico Pasante de Servicio Social en el Centro de Ciencias de la Salud de la Universidad Autónoma de Aguascalientes mopa9417@gmail.com

** Ma. del Carmen Terrones Saldívar. Dra. en Ciencias. Profesora Investigadora de Tiempo Completo del Centro de Ciencias de la Salud de la Universidad Autónoma de Aguascalientes. mcterron@correo.uaa.mx

Fecha de recibido: 25 de septiembre 2019

Fecha de aceptación: 6 de diciembre 2019

Correspondencia: Dra Ma del Carmen Terrones Saldívar. Departamento de Medicina. Edificio 107 planta alta. Centro de Ciencias de la Salud. Avenida Universidad 940, Ciudad Universitaria. Código postal 20131. Aguascalientes, Ags., México. Teléfono 9107400 extensión 53009. Correo electrónico mcterron@correo.uaa.mx 


\section{Introducción}

Las malformaciones congénitas $(M C)$ son anormalidades estructurales o funcionales de un órgano o parte de él o zonas más extensas del organismo que están presentes desde el nacimiento y se codifican según la CIE-10 en Q00-Q99. ${ }^{1}$ Pueden ser mayores o menores, entendiendo a las primeras como aquellas que generan una limitación biopsicosocial en el individuo. ${ }^{2}$ En algunas situaciones se agrupan como síndromes, secuencias o asociaciones; en los primeros se presentan múltiples anomalías generadas por una misma causa; en las segundas, las alteraciones se desarrollan en sucesión como resultado de una lesión inicial. Finalmente, las asociaciones representan un grupo de afectaciones que producen frecuentemente problemas particulares pero que no tienen ninguna relación etiológica. ${ }^{3}$

La Organización Mundial de la Salud (OMS) estima una prevalencia internacional homogénea en recién nacidos vivos entre 3-5\% y en muertes fetales de 15-20\%., ${ }^{4,5}$ Además, afectan a 1 de cada 33 lactantes, lo cual ocasiona 3.2 millones de discapacidades al año y 303,000 muertes neonatales, posicionándolas como la segunda causa de muerte en menores de 28 días y de 5 años en las Américas, y como la primera causa en Europa. Asimismo, el 94\% de las $M C$ graves se reportan en países de ingresos bajos y medios, donde las mujeres frecuentemente no tienen acceso a los nutrientes necesarios, control prenatal adecuado o presentan mayor exposición a agentes o factores como la infección y el alcohol. ${ }^{6,7}$ La prevalencia de malformaciones congénitas en países latinoamericanos reportada por el Estudio Colaborativo Latinoamericano de Malformaciones Congénitas (ECLAMC) es del 3.4\%.8 En países como Chile y Cuba se ha demostrado una tasa de 386.6/10,000 nacimientos y de $89 / 10,000$ recién nacidos vivos, respectivamente. ${ }^{9,10}$

Para México, la prevalencia de malformaciones congénitas ha sido del $0.7 \%$ en nacidos vivos y del $8.3 \%$ en muertes fetales con una tasa de 77.3/10,000 nacimientos, ${ }^{11}$ ubicando al estado de Aguascalientes con la tasa más alta (187.4/10, 000 nacimientos). ${ }^{5}$ Otros reportes en México han indicado una prevalencia de $M C$ del $0.95 \%$ en Sinaloa. ${ }^{12}$

A pesar de que la mortalidad infantil en menores de un año en México ha disminuido $61.5 \%$ en los últimos 30 años, las MC como causa de muerte han incrementado $28.7 \%$ posicionándola como la segunda causa de mortalidad infantil. ${ }^{13}$ En Chile también ha ocurrido lo anterior, ya que el $35.8 \%$ de las muertes infantiles se atribuyeron a MC mostrándose por encima de las tasas para otros países Europeos y reflejando un retraso en el abordaje de este problema de salud pública en Latinoamérica. ${ }^{14,15}$

\section{Génesis de las malformaciones congénitas}

La etiología de las MC se atribuye mayormente a factores desconocidos (65-75\%), anomalías genéticas (20-25\%), infección fetal 
(3-5\%), enfermedad materna (2-4\%) y drogas durante el embarazo $(<1 \%)$. Entre las causas desconocidas podría presentarse la combinación de múltiples factores hereditarios, teratógenos y afectaciones del microambiente fetal. Además, se ha formulado un modelo en el que hay una predisposición genética que permite un bajo umbral en la capacidad para autorregular el patrón de desarrollo normal ante múltiples exposiciones ambientales y la forma en que el producto interactúa con el agente, la etapa del desarrollo embrionario en la que interviene el teratógeno o factor de riesgo y la dosis administrada o la agregación e interacción de varios agentes en una misma emisión. Finalmente, se define como teratógeno a los factores físicos o químicos ambientales que generan un efecto adverso sobre el embrión, mientras que los factores de riesgo son aquellas condiciones que directa o indirectamente contribuyen a que se modifique el desarrollo normal del feto, el estado materno o ambos. 6, 16-19

\section{Factores asociados al desarrollo de malformaciones congénitas}

Los factores sociodemográficos identificados son la edad de los padres al momento de la concepción; número y espaciamiento de embarazos; rasgos raciales; escolaridad de los padres; consanguinidad y nivel socioeconómico bajo. ${ }^{20}$ Lo último por resultado directo de la desinformación en los padres durante la atención prenatal o un efecto indirecto de la región de residencia (falta de acceso a hospitales, regiones de mayor riesgo, violencia, contaminación, entre otros) o mayor grado de exposición materna al alcohol, tabaco y otras drogas, así como una alimentación deficiente en nutrientes. ${ }^{21}$

Los factores ambientales asociados a MC son: exposición a plaguicidas o agroquímicos, exposición a arsénico; ocupación de la madre, vivienda menor a un kilómetro de campos agrícolas; uso doméstico de plaguicidas, lavado de ropa contaminada con productos agroquímicos y trabajo de la madre en actividad agrícola. ${ }^{22,23}$

Sobre los factores clínico-patológicos se ha documentado que madres con diabetes mellitus preconcepcional y gestacional, enfermedad hipertensiva en el embarazo, asma bronquial, antecedentes patológicos familiares de $M C$, restricción de crecimiento intrauterino, antecedentes obstétricos de amenaza de aborto o abortos previos se han asociado con mayor frecuencia a defectos congénitos. ${ }^{20,} 22$ Además, se resalta la trascendencia de la diabetes mellitus en la generación de malformaciones congénitas debido a la gran prevalencia de esta enfermedad en población mexicana. ${ }^{24}$ La asociación de HTAS y MC aún es debatida, pero se ha presentado relación entre pre-eclampsia o hipertensión gestacional y el desarrollo de hipospadias y entre HTAS crónica con atresia esofágica. ${ }^{25,} 26$

Es importante mencionar que si bien existen infecciones asociadas a complicaciones perinatales maternas y fetales, estos defectos detectados en el nacimiento no son clasificados como malformaciones congénitas, sino que forman parte de las manifestaciones 
causadas por el agente infeccioso y no se incluyen en la sección Q00-Q99 del manual CIE-10.27

Finalmente, en el grupo de medicamentos asociados al desarrollo de $M C$ están las benzodiacepinas, fármacos inhibidores del sistema renina-angiotensina-aldosterona como los IECA y los ARAII, ácido retinoico, inhibidores de la dihidrofolato reductasa (metotrexate, pirimetamina, triamtereno, trimetropim), modificadores del metabolismo de folatos (carbamazepina, fenobarbital y fenitoína), ácido valproico y las estatinas, por lo que se recomienda suspenderlos o evitarlos durante la gestación. ${ }^{28}$

\section{Intervenciones de salud pública en la prevención de malformaciones congénitas}

Las intervenciones que la salud pública implementa han repercutido notoriamente en la modificación de factores de riesgo de tipo ambiental. Un ejemplo claro fue con la evaluación de la suplementación dietética periconcepcional con ácido fólico en los 70 y la demostración de su efectividad con dos ensayos controlados aleatorizados en la década de los $90 .^{29}$ Sin embargo, hasta la aplicación de medidas reglamentarias de uso de suplementos de ácido fólico así como la adición de este componente en harina de trigo en países como EUA y Chile, se presentó una reducción significativa de los defectos del tubo neural. 20, 29, 30

Aunque las estadísticas sobre la prevalencia de $M C$ nacionales son variadas, no hay reportes previos publicados sobre este fenómeno en el estado de Aguascalientes y su distribución en los 11 municipios que lo conforman. Por lo anterior, el presente estudio busca contribuir a la comprensión del comportamiento epidemiológico de dicho suceso para posteriormente realizar intervenciones en la detección de factores, prevención, diagnóstico e injerencias en el manejo de la mortalidad infantil y discapacidades atribuibles a MC.

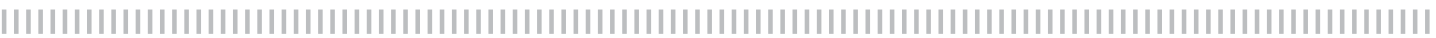

\section{Material y métodos}

Se realizó un estudio observacional, retrospectivo, transversal. Se incluyeron a los recién nacidos vivos entre las semanas 22 y 42 de gestación o con peso mayor o igual a $500 \mathrm{~g}$ con diagnósticos de una o más malformaciones congénitas, otras lesiones perinatales o aparentemente sanos, cuya madre tuviera residencia habitual en el estado de Aguascalientes y registrados en el formato de Certificado de nacimiento durante enero de 2008 a diciembre de 2017 con acceso en el portal de la Direc- ción General de Información en Salud, de la Secretaria de Salud de los Estados Unidos Mexicanos. * Lo anterior debido a que actualmente este instrumento tiene cobertura nacional y acceso libre para su consulta mediante el Sistema Nacional de Información en Salud (SINAIS), Dirección General de Información en Salud (DGIS) de la Secretaría de Salubridad y Asistencia (SSA). Es relevante indicar que dicha información recabada, recopilada y difundida a nivel nacional ha sido avalada por el Instituto Nacional de Estadística y Geografía

* Con dirección: http://www.dgis.salud.gob.mx/contenidos/basesdedatos/estandar_gobmx.html. 
(INEGI) en la publicación del Diario Oficial de la Federación (DOF) del 23 de mayo de 2013, mencionando que los indicadores de salud sobre eventos perinatales deben obtenerse con datos del SINAC. ${ }^{31}$

Para el análisis estadístico se utilizó el programa SPSS 25. Los resultados obtenidos de las variables cualitativas se compa-

\section{| | | | | | | | | | | | | | | | | | | | | | | | | | | | | | | | | | | | | | | | | | | | | | | | | | | | | | | | | | | | | | | | | | | | | | | | | | | | | | | | | | | | | | | | | | | | | | | | | | | | | | | | | | | | | | | | | | |}

\section{Resultados}

Se integró una base de datos con 267,489 nacidos vivos durante 2008-2017, de los cuales se eliminaron $13,372(5 \%)$ debido a información faltante o mal llenado del diagnóstico en el certificado. Se identificó una prevalencia de $2.1 \%(5,595)$ con una tasa de 209.1 por cada 10,000 nacidos vivos. El resto fue codificado como sano $(68.6 \%)$, problemas del recién nacido vivo y muerto relacionados con factores maternos y complicaciones del embarazo o trabajo de parto $(24.3 \%)$ y mal codificados o ausencia de datos (5\%). Se muestra la prevalencia de $M C$ por año en la figura 1, resaltando que la más alta ocurrió en 2016. Las malformaciones del sistema osteomuscular fueron las más frecuentes (39.3\%), raron mediante la fórmula de $\mathrm{X}^{2}$, mientras que para las variables identificadas como cuantitativas se utilizó la fórmula de $T$ de Student. Se realizó un análisis estadístico de las variables con un rango de confianza del $95 \%(\alpha=0.95)$, un margen de error del $5 \%(0.05)$ y una potencia del $80 \%(0.80)$.

siguiéndole las malformaciones de los órganos genitales $(19 \%)$ y las malformaciones congénitas del ojo, del oído, de la cara y del cuello (12\%) (figura 2 y tabla 1). De manera individual, las 10 malformaciones congénitas más frecuentes fueron testículo no descendido (14.2\%), deformidades congénitas de los pies $(11.5 \%)$, deformidades congénitas de la cadera (11.2\%), otras malformaciones congénitas del oído $(10.5 \%)$, fisura del paladar con o sin labio leporino $(5.6 \%)$, síndrome de Down $(4.3 \%)$, polidactilia $(4.2 \%)$, malformaciones congénitas del sistema osteomuscular, no clasificadas en otra parte $(3.0 \%)$, otras malformaciones congénitas no clasificadas en otra parte $(2.9 \%)$ e hipospadias $(2.7 \%)$ (tabla 2).

Prevalencia de malformaciones congénitas por año de ocurrencia

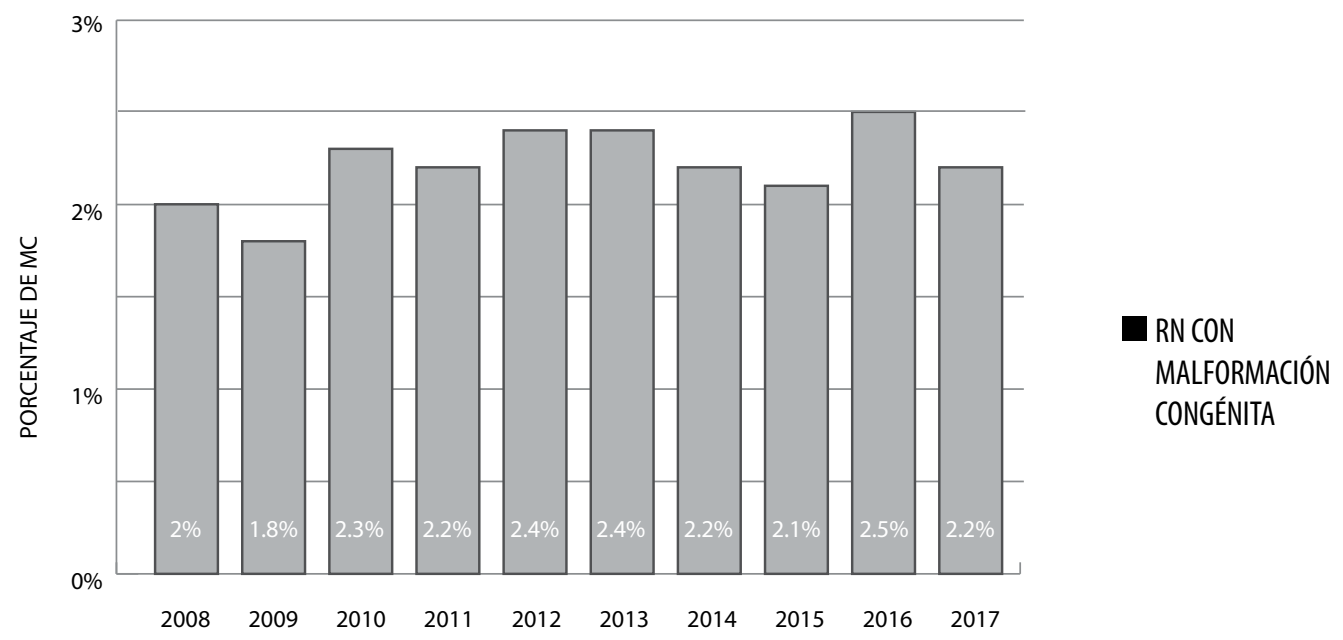

Figura 1. Se muestra la prevalencia de MC por año de ocurrencia, la cual, aunque no ha superado el 3\%, sí ha tenido variaciones estadísticamente significativas p<0.01, con su mayor frecuencia en 2016. 


\section{Distribución de MC por aparatos y sistemas}

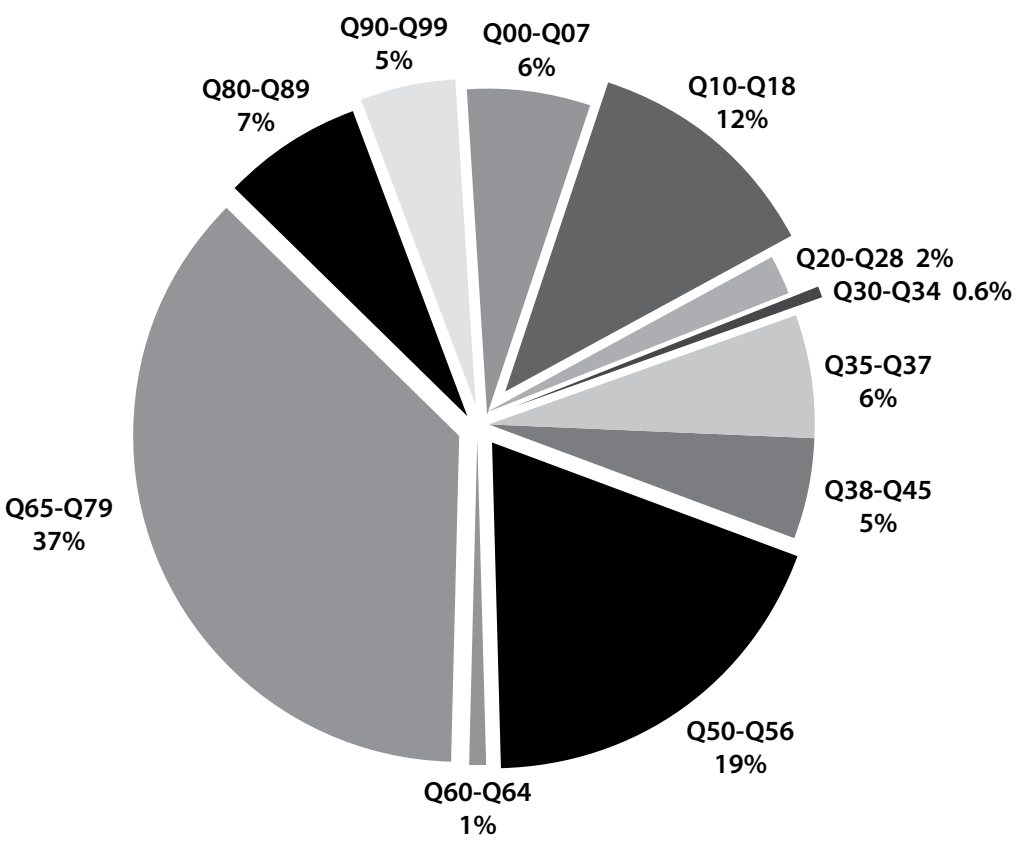

Figura 2. Se muestran las malformaciones congénitas distribuidas por aparatos y sistemas codificados mediante la CIE-10 capitulo XVII, siendo las MC del sistema osteomuscular (Q65-Q79), de órganos genitales (Q50-Q56), del ojo, del oído, de la cara y del cuello (Q10-Q18), otras malformaciones (Q80-Q89) y malformaciones del paladar y labio leporino (Q35-Q37) las más frecuentes. Además, se identifican a las $M C$ del sistema respiratorio (Q30-Q34) como las menos frecuentes.

\section{Tabla I}

\section{Se muestran las MC agrupadas por aparatos y sistemas, señalando la tasa de prevalencia por cada 10,000 nacidos vivos}

\begin{tabular}{|llll|}
\hline Malformaciones congénitas, deformidades y anomalías cromosómicas (Q00-Q99) & $\mathrm{N}$ & $\%$ & Tasa $^{*}$ \\
\hline Malformaciones congénitas del sistema nervioso (Q00-Q07) & 306 & $5.5 \%$ & 11.4 \\
\hline Malformaciones congénitas del ojo, del oído, de la cara y del cuello (Q10-Q18) & 663 & $12 \%$ & 24.7 \\
\hline Malformaciones congénitas del sistema circulatorio (Q20-Q28) & 88 & $1.6 \%$ & 3.2 \\
\hline Malformaciones congénitas del sistema respiratorio (Q30-Q34) & 32 & $0.6 \%$ & 1.1 \\
\hline Fisura del paladar y labio leporino (Q35-Q37) & 312 & $5.6 \%$ & 11.6 \\
\hline Otras malformaciones congénitas del sistema digestivo (Q38-Q45) & 255 & $4.6 \%$ & 9.5 \\
\hline Malformaciones congénitas de los órganos genitales (Q50-Q56) & 1064 & $19 \%$ & 39.7 \\
\hline Malformaciones congénitas del sistema urinario (Q60-Q64) & 54 & $1 \%$ & 2 \\
\hline Malformaciones y deformidades congénitas del sistema osteomuscular (Q65-Q79) & 2193 & $36.2 \%$ & 81.9 \\
\hline Otras malformaciones congénitas (Q80-Q89) & 361 & $6.5 \%$ & 13.4 \\
\hline Anomalías cromosómicas, no clasificadas en otra parte (Q90-Q99) & 257 & $4.6 \%$ & 9.6 \\
\hline
\end{tabular}




\section{Tabla 2}

Se muestran las 10 MC individuales más frecuentes y la tasa de prevalencia de las mismas por 10,000 nacidos vivos. **Aunque no se ubican entre las MC más frecuentes, los defectos del tubo neural se muestran en esta tabla debido a que tienen una vigilancia epidemiológica estricta

\begin{tabular}{|llll|}
\hline Malformaciones congénitas, deformidades y anomalías cromosómicas (Q00-Q99) & $\mathrm{n}$ & $\%$ & Tasa $^{*}$ \\
\hline Testículo no descendido & 796 & $14.2 \%$ & 29.8 \\
\hline Deformidades congénitas de los pies & 642 & $11.5 \%$ & 24 \\
\hline Deformidades congénitas de la cadera & 620 & $11.1 \%$ & 23.2 \\
\hline Otras malformaciones congénitas del oído & 589 & $10.5 \%$ & 22 \\
\hline Fisura del paladar con o sin labio leporino & 312 & $5.6 \%$ & 11.7 \\
\hline Síndrome de Down & 240 & $4.3 \%$ & 8.9 \\
\hline Polidactilia & 236 & $4.2 \%$ & 8.8 \\
\hline MC del sistema osteomuscular, no clasificadas en otra parte & 166 & $3 \%$ & 6.2 \\
\hline Otras malformaciones congénitas no clasificadas en otra parte & 160 & $2.9 \%$ & 5.9 \\
\hline Hipospadias & 149 & $2.7 \%$ & 5.6 \\
\hline Anencefalia y malformaciones congénitas similares** & 18 & $0.3 \%$ & 0.7 \\
\hline Encefalocele** & 8 & $0.1 \%$ & 0.3 \\
\hline Espina bífida** $^{* *}$ & $1.7 \%$ & 3.5 \\
\hline Otras malformaciones congénitas de la médula espinal** & 94 & $0.07 \%$ & 0.1 \\
\hline Defectos del cierre del tubo neural** & 4 & $2.2 \%$ & 6.7 \\
\hline
\end{tabular}

\section{Tabla 3}

Tasa de prevalencia por 10,000 nacidos vivos. Se destaca que la prevalencia y tasas de prevalencia más altas se encuentran en los municipios del suroeste.

Malformaciones congénitas por municipio en el periodo 2008-2017

\begin{tabular}{|lccc|} 
Municipio & Número de $M C$ & $\%$ & Tasa \\
\hline El Llano & 113 & 2.9 & 290.1 \\
\hline Aguascalientes & 3984 & 2.4 & 236.5 \\
\hline San Fco. de los Romo & 171 & 2.2 & 218.6 \\
\hline Jesús María & 436 & 2.1 & 214.9 \\
\hline Asientos & 223 & 2.1 & 210.6 \\
\hline Calvillo & 153 & 1.3 & 131.2 \\
\hline Tepezalá & 83 & 1.8 & 181.5 \\
\hline Rincón de Romos & 201 & 1.7 & 169.4 \\
\hline San José de Gracia & 32 & 1.6 & 159.6 \\
\hline Pabellón & 143 & 1.5 & 152.1 \\
\hline Cosío & 51 & 0.9 & 145.7 \\
\hline
\end{tabular}

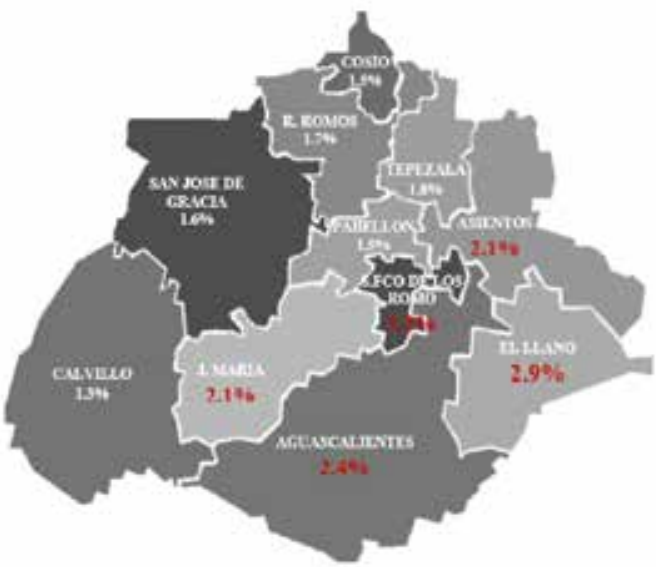

Figura 3. Se ilustra la distribución de las malformaciones congénitas en los municipios que conforman al estado de Aguascalientes según el lugar de residencia habitual de la madre. Se destacan las mayores prevalencias en los municipios del suroeste del estado. 
Por municipio de residencia habitual de la madre, los municipios El Llano y Aguascalientes obtuvieron las prevalencias más altas de $2.9 \%$ y $2.4 \%$, respectivamente, con una tasa mayor a la media del estado de 290.1 para el municipio de El Llano y de
236.5 para el municipio de Aguascalientes. Finalmente, resulta importante señalar que la diferencia en la prevalencia de MC por municipio de residencia de la madre presentó asociación estadísticamente significativa $(p<0.01)$.

\section{| | | | | | | | | | | | | | | | | | | | | | | | | | | | | | | | | | | | | | | | | | | | | | | | | | | | | | | | | | | | | | | | | | | | | | | | | | | | | | | | | | | | | | | | | | | | | | | | | | | | | | |}

\section{Discusión}

La prevalencia de malformaciones congénitas de $2.1 \%$ resultó menor a los estándares internacionales emitidos por la OMS de entre $3-5 \%$, así como al 3.4\% para la población latinoamericana publicada por el equipo $\mathrm{ECLAMC}^{8}$ al $3.9 \%$ de $\mathrm{MC}$ en recién nacidos vivos en Chile durante 2001$2010^{10}$ y ligeramente menor al $2.39 \%$ publicado para series en Sinaloa. ${ }^{32}$ No obstante, al comparar los resultados con series nacionales previas se identifica un frecuencia mayor a lo reportado por Navarrete y Canún de $0.7 \%$ a nivel nacional durante 2008-2013. Además, la tasa de prevalencia obtenida fue de 209.1/10,000 RNV siendo superior a los resultados previos en Guadalajara, CDMX y Monterrey, así como la media nacional de 77.1/10,000 nacimientos para 2008-2013. 5, 11,33 Aunque anteriormente se había ubicado al estado de Aguascalientes como la entidad con la tasa de MC más alta en la presente serie se identificó una tasa superior a la cifra calculada $\left(187.4 / 10,000\right.$ nacimientos). ${ }^{5}$ Se resalta que debe interpretarse con cautela la variación en la ocurrencia de MC por año debido a que el programa de Certificado de Nacimiento y Certificado de muerte fetal con cobertura nacional y desglose por el SINAC fue implementado a partir del año 2007, por lo que el correcto llenado y reporte de los eventos perinatales pudiesen presentar un subregistro en los primeros años.

Las MC del sistema osteomuscular fueron las más frecuentes (36.2\%) resultando semejante a lo indicado por Vázquez para población cubana y por Navarrete-Canún para la mexicana ubicando a las MC del osteomuscular como las más frecuentes $\left(40.5 \%\right.$ y $37.1 \%$, respectivamente).$^{5,9} \mathrm{Sin}$ embargo, las MC cardiovasculares (1.6\%) en la presente serie se ubicaron entre las tres menos frecuentes discrepando con lo publicado por Russell en EUA en cuya serie se ubicaron como las más frecuentes. También, diverge de la prevalencia referida en población mexicana de $16.2 \%$ y del $16.9 \%$ para población Chilena. ${ }^{10,30,32}$

Se encontró que la criptorquidia (testículo no descendido) representó la MC más frecuente, siguiéndole las deformidades congénitas de los pies (11.5\%) y deformidades congénitas de la cadera (11.2\%) concordando con lo reportado en series mexicanas previas respecto a las tres principales causas de malformaciones congénitas, aunque ubicando a las deformidades congénitas de los pies como las más frecuentes con $10.8 \% .^{33}$

Respecto a las malformaciones cromosómicas, el síndrome de Down se ubicó como la más frecuente siendo afín a las series mexicanas e internacionales publicadas. Sin embargo, la tasa obtenida (8.9/10,000 RNV) difiere de lo reportado para México de 3.7 y 3.55 para el periodo 2008-2013, respectivamente. También, resulta discordante de la alta tasa publicada en Chile entre 2001-2010 de $25 / 10,000$ RN y del 13.8/10,000 RNV en población hispana residente en EUA entre 2004-2006. 5, 10, 30, 33

Los defectos del tubo neural fueron el $2.2 \%$ de las $M C$, con una tasa de $4.6 / 10,000$ RNV y no manifestaron asociación por el año de ocurrencia. La anen- 
cefalia exhibió un comportamiento similar a lo reportado a nivel nacional por $\mathrm{Na}$ varrete para el periodo de 2009-2010, y discretamente superior a la serie de Canún para 2013 (0.7 vs 0.4/ 10,000 RNV). Sin embargo, fue menor al 2.03 en población hispana en EUA entre 1999-2007, quizá por ser una comparativa temporal distinta y un periodo en el que la fortificación en población hispana estuviese reducida. Para la espina bífida, los hallazgos difieren de la tasa nacional encontrada previamente de 1.15-1.31 en RNV, pero semejante al reporte de Canún para Aguascalientes entre 2008-2012 (3.7/10,000 RNV). ${ }^{5,11,30}$

\section{Conclusiones}

La prevalencia de $M C$ en el estado de Aguascalientes es mayor que la prevalencia nacional. Se destaca la distribución principal en los municipios del suroeste del estado, por lo que se deberán identificar los factores y así realizar intervenciones regulatorias ambientales o clínicas. Además, se debe realizar el diseño de medidas de rehabilitación e integración social para las personas portadoras de algún defecto congénito, ya que el número de nacimientos ha sido constante (entre 25,000$27,000)$ y, considerando la tasa de $M C$ por año de 565 recién nacidos vivos para el 2030, el estado se afrontará a 6210 casos nuevos. Esto alude a la importancia de aplicar medidas de salud pública para la detección y modificación de factores asociados y, así, prevenir los nuevos casos proyectados para reducir los gastos requeridos para la atención de las complicaciones asociadas a MC, amortiguando la mortalidad infantil y asegurar un desarrollo intrauterino óptimo.

Finalmente, hay que destacar la importancia de la capacitación del personal en el diagnóstico de MC menores y mayores, así como un correcto llenado del certificado de nacimiento que incluya el reporte de aquellas malformaciones diagnosticadas durante el periodo prenatal y no sólo las malformaciones congénitas externas, factor relevante en el predominio de los defectos congénitos del sistema osteomuscular.

\section{Bibliografía}

1.- OMS/CDC/ICBDSR. Vigilancia de anomalías congénitas. Atlas de algunos defectos congénitos. Ginebra: Organización Mundial de la Salud; 2015.

2.- Smith C. Donnai D. Human malformations. En: Rimoin DL, Connor JM, Pyeritz RE, Korf BR. Emery and Rimoin's Principles and Practice of Medical Genetics. 6th Edition. Vol. 1. New York: Churchill Livingstone. 2013; 488-500.

3.- Cunninham F. Leveno K. Bloom S. Hauth J. Rouse D. Spong C. Genética. Williams Obstetricia. México: McGraw-Hill, Interamericana. 23a edición. 2011. pp 287-311.

4.- Flores G. Pérez T. Pérez M. Malformaciones Congénitas Diagnosticadas en un Hospital General. Revisión de cuatro años. Acta Pediatr Mex. 2011; 32(2):101-106.

5.-Navarrete, H. Canún, S. Reyes, P. Sierra, R. Valdés, H.
Prevalencia de malformaciones congénitas registradas en el certificado de nacimiento y de muerte fetal. México, 2009-2010. Bol Med Hosp Infant Mex. 2013; 70(6):499-505.

6.-Organización Mundial De La Salud. Defectos congénitos. $63^{a}$ Asamblea Mundial de la Salud. A63/10. 2010. Consultada en: http://apps.who. int/gb/ebwha/pdf_files/WHA63/A63_10-sp. pdf?ua $=1 \& u a=1 \& u a=1$. (Fecha de consulta: 2001-2019).

7.-Organización Mundial de la Salud. Anomalías congénitas. 2016. https://www.who.int/es/news-room/ fact-sheets/detail/congenital-anomalies. Ultima vista: 13-2-19.

8.-Matovelle C. Matovelle P. Martinez F. Cordova F. Estudio Descriptivo: Frecuencia de Malformaciones Congenitas en Paciente Pediatricos del Hospital "Jose Carrasco Arteaga". Rev Med HJCA. 2015; 7(3):249-253. 
9. Vázquez V. Torres C. Dueñas A. Vázquez G. Díaz D. López R. Malformaciones congénitas en recién nacidos vivos. Medisur. 2013;12(1): 42-50.

10 Nazer J. Cifuentes L. Prevalencia al nacimiento de malformaciones congénitas en las maternidades chilenas participantes en el ECLAMC en el período 2001-2010. Rev Med Chile 2014; 142: 1150-1156.

11 Canún S. Navarrete E. Aldelmo E. Reyes A. Valdés Hernández J. Prevalencia de malformaciones congénitas de herencia multifactorial de acuerdo con los certificados de nacimiento y muerte fetal. México, 2008-2012. Bol Med Hosp Infant Mex. 2015; 72(4):290-294.

12 Ramos E. Monzón A. Dautt J. Frecuencia de malformaciones congénitas en recién nacidos del Hospital de la Mujer. Arch Salud Sin. 2011; 5(4):101-105.

13 Fernández S. Gutiérrez G. Viguri R. Principales causas de mortalidad infantil en México: tendencias recientes. Bol Med Hosp Infant Mex. 2012; 69: 144148.

14. Dipierri J. Acevedo N. Bronberg R. Mortalidad infantil por malformaciones congénitas en Chile: análisis temporal y espacial, 1997-2011. Rev Panam Salud Pública. 2015; 38(5):380-387.

15 Gómez A. Rascón R. La mortalidad infantil por malformaciones congénitas en México: un problema de oportunidad y acceso al tratamiento. Rev Panam Salud Pública 2008; 24(5): 297-303.

16 Rojas M. Walker L. Malformaciones congénitas: aspectos generales y genéticos. Int. J. Morphol 2012. 30(4): 1256-1265.

17 Acosta C. Mullings R. Caracterización de malformaciones congénitas en recién nacidos vivos. MediSur. 2015; 13: 375-382.

18 Ristivojevi? A. Luki? D. Katani?. Du?anka D. Jovanovi? P. Epidemiology and structure of congenital anomalies of the newborns in the region of Novi Sad (Vojvodina, Serbia) in 1996 and 2006. Vojnosanit Pregl. 2016; 73(5):442-448.

19 Dolk H. Loane M. Garne E. The prevalence of congenital anomalies in Europe. Posada M. Groft S. Rare Diseases Epidemiology, Advances in Experimental Medicine and Biology. Reino Unido. Springer Science Business Media. 2010; 349-364.

20 Canals A. Cavada G. Nazer J. Factores de riesgo de ocurrencia y gravedad de malformaciones congénitas. Rev Med Chile 2014; 142:1431-143.

21 Pawluk M. Campaña H. Gili J. Comas B, Giménez L. Villalba M. Scala S. Poletta F. López J. Adverse social determinants and risk for congenital anomalies. Arch Argent Pediatr. 2014; 112(3):215-223.
22 Concepción L. Benítez S. Factores de riesgo prenatales y su asociación a malformaciones congénitas en un Hospital Universitario de Referencia. Pediatr. 2018; 45(1):08-16.

23 Lin S. Herdt M. Chapman B. Munsie J. Olshan A. Druschel C. Maternal occupation and the risk of major birth defects: A follow-up analysis from the $\mathrm{Na}$ tional Birth Defects Prevention Study. International Journal of Hygiene and Environmental Health. 2013; 216:317-323.

24 Cooper O. Hernández D. Arbogast G. y cols. Major Congenital Malformations after First Trimester Exposure to ACE Inhibitors. The New England Journal of Medicine. 2006; 354. (23):2443-2451.

25 Van G. Van B. Louik C. Werler M. Roeleveld N. Mitchell A. Maternal Hypertensive Disorders, Antihypertensive medication use, and the risk of birth defects: a case- control study. Royal College of Obstetrician and Gynecologists. 2014; 122(7):1002-1009.

26 Van Z. Werler M. Browne M. y cols. Maternal Hypertension, Medication Use, Hypospadias in the National Birth Defect Prevention Study. Obstetrics and Gynecology. 2014; 123(2):309-317.

$27 \mathrm{Neu}$ N. Duchon J. Zachariah P. TORCH Infections. Clin Perinatol. 2015; 42 (1):77-103.

28 Harris B. Bishop K. Kemeny H. Walker J. Rhee E. KuIler J. Risk Factors for Birth Defects. Obstet Gynecol Surv. 2017; 72(2):123-135

29 Czeizel A. Dudas I. Preventionsof. The First Occurrence Of Neural-Tube Defects By Periconceptional Vitamin Supplementation. The New Eng J of Med. 1992; 327(26):1832-1835.

30 Russell S. The prevalence of selected major birth defects in the United States. Seminars in perinatology. 2017; 41 (6): 338-334.

31 Secretaría de Salud. Manual de Llenado del Certificado de Nacimiento. Subsistema de Información sobre Nacimientos (SINAC). Primera Edición. Dirección General de Información en Salud. Secretaría de Salud. México, 2015. pp-20. Revisado en: http://www. dgis.salud.gob.mx/descargas/pdf/Manual_Instalacion_Operacion_SINAC.pdf (Fecha de última vista: (2-04-19)

32 Calderón A. Rojas M. Dehesa E. Prevalencia de malformaciones congénitas detectadas al nacimiento en un hospital de segundo nivel en Sinaloa. Acta Pediátrica Mex. 2017; 38(6):363-370.

33 Navarrete E. Canún S. Valdés J. Reyes A. Malformaciones congénitas al nacimiento: México, 20082013. Bol Med Hosp Infant Mex. 2017; 74(4):301 308. 